\title{
Epidemiological analysis of passive surveillance data on foot and mouth disease occurrence in Nakuru County, Kenya
}

\begin{abstract}
Introduction: Epidemiological analysis of passive surveillance data of animal diseases holds great potential in uncovering disease trends both temporally and spatially and flaws if any on the passive surveillance mechanisms in place. A study was conducted to investigate FMD occurrence in a county in Kenya.

Methodology: Data from 171 samples was obtained from the National Foot and Mouth Disease (FMD) laboratory covering the isolation of the Foot and Mouth disease Viruses in Nakuru County for the period 2010-2016. The samples were self-submitted by community animal health workers and farmers as well as from outbreak response teams

Results: A total of $107 / 171(69.3 \%)$ of the samples were positive for FMDV with outbreaks occurring every year under study. Four serotypes (A, O, SAT -1 and SAT-2) were isolated with outbreaks of different serotypes occurring simultaneously. There was weak evidence of seasonality with the highest relative risk of sample submission being observed in March $(4.75 ; 2.84-7.92,95 \%$ CI) and September $(6.5 ; 3.91-10.80$ $95 \% \mathrm{CI}$ ), the final months of the dry seasons. Sample positivity did not show seasonal variation. However, there was significantly more sample collection during the dry season than in the wet season $(\mathrm{p}=0.02)$.

Conclusion: FMD is still a major concern in Nakuru County with different serotypes in circulation. The reason for seasonality should be investigated including effect of livestock movements in FMD outbreaks in Kenya. Passive surveillance data is useful in determining temporal patterns in disease occurrence. However intermittent active surveillance during off seasons might help reduce sampling bias.
\end{abstract}

Volume 6 Issue 3 - 2017

\author{
Machira DN, Kitala PM \\ Masters Student, University of Nairobi, Kenya
}

Correspondence: Machira DN, Masters Student, The University of Nairobi, Kenya, Tel +254 704670 347, Email machira.dickson@gmail.com

Received: October 23, 2017 | Published: December 28, 2017
Abbreviations: FMD, foot and mouth disease; FMDV, foot and mouth disease virus; SAT, southern african territories; IRR, incidence risk rates

\section{Introduction}

Foot and Mouth Disease (FMD) is a viral disease affecting clovenhoofed animals. The causative pathogen, the Foot and Mouth Disease Virus (FMDV) is a single stranded positive sense virus belonging to the family picornaviridae and genus aphthovirus. ${ }^{1}$ The disease is endemic in Kenya. ${ }^{2}$ Though the disease has a low case fatality rate, the high morbidity rate and impact on productivity means the disease can have a huge impact on dairy farming. ${ }^{3}$ Foot and mouth disease viruses occur in serotypes that have no crossover protection. These are $\mathrm{O}, \mathrm{A}$, C, Southern African Territories (SAT)-1, SAT 2, SAT 3 and Asia 1. In Kenya, outbreaks of O, A, C, SAT 1 and SAT 2 have been previously reported ${ }^{4}$ although serotype $\mathrm{C}$ has not been detected worldwide since 2004 and may no longer be present. The National foot and mouth disease laboratory in Kenya is a government laboratory that is mandated with FMD surveillance in Kenya. The laboratory conducts both active and passive surveillance in the country. As part of its passive surveillance system the lab receives self-submitted samples from farmers and health practitioners on suspected FMD cases as well as samples it collects as part of its outbreak response activities. The laboratory has capacity to do various serological and molecular tests to identify FMD and its serotypes. ${ }^{5}$
Epidemiological analysis of accumulated passive surveillance data of animal diseases holds great potential in uncovering disease trends both temporally and spatially and flaws if any on the passive surveillance mechanisms in place. In addition, it may expose new knowledge gaps to inform research and provide critical information for formulation of control programs. Analysis of passive surveillance data has been done before to investigate trends in temporal and spatial distribution of disease. Naumova and colleagues found evidence of seasonality in Giardiasis and cryptosporidiosis in America using passive surveillance data. ${ }^{6}$ compared active and passive surveillance methods in detection of cerebrovascular disease. Active surveillance had a lower positive predictive than passive surveillance. In addition passive surveillance systems are usually less costly. The aim of this study was therefore to investigate FMD occurrence in Nakuru County, Kenya using available passive surveillance data.

\section{Methodology}

The Kenya National Foot and Mouth Disease Laboratories receives samples submitted from outbreak areas on a continuous basis. The laboratory has capacity for serotyping and genotyping the samples using molecular and serology based tests. ${ }^{6}$ FMD suspect samples are submitted to the laboratory for FMDV detection and serotyping. The samples are self-submitted by the cattle farmers from cattle suspected to have foot and Mouth Disease. In addition some samples are collected as part of outbreak response to suspected 
outbreaks of FMD in the country. Data on the date the sample was submitted and location information is collected at sample submission. Detection and serotyping of FMDV in the samples is conducted using NSP-ELISA according to methods reported before. ${ }^{7}$ Sample collection data, positivity and serotypes detected from the samples is then stored in a database. Data was extracted from the National FMD laboratories' database on all samples submitted to the laboratory from Nakuru County for the years 2010 to 2016. The data was extracted in Microsoft excel form and imported into STATA version 13 (Stata Corp., USA) for cleaning and analysis. Exploratory descriptive analysis was conducted on to determine frequency distribution on year, location and serotypes detected. Generalized linear models were fitted on count data of samples submitted per month to determine if

Table I Serotype distribution among the samples seasonality predicted sample submission.

\section{Results}

Data from a total of 171 samples was available from the National FMD laboratory database. The data covered a period of 6years, from 2010-2016. The number of samples collected were highest in $2013(34 / 171(19.9 \%))$ and lowest in $2016(13 / 171(7.6 \%))$ A total of $109 / 171(63.7 \%)$ samples tested positive for at least 1 serotype of FMDV. Serotype O was the most isolated with $25.15 \%$ of the samples being positive. This was followed by SAT-2, A and SAT-1 with $17.5 \%, 11.7 \%$ and $9.4 \%$ positivity respectively. No other serotype was detected and no sample had more than one serotype detected in Table 1.

\begin{tabular}{|c|c|c|c|c|c|}
\hline \multicolumn{6}{|l|}{ Proportion(\%) } \\
\hline $\begin{array}{l}\text { Year(Number of Samples } \\
\text { Collected) }\end{array}$ & A & 0 & SAT-I & SAT-2 & Overall positivity \\
\hline $2010(29 / 171)$ & 0 & 6/29(20.7) & $7 / 29(24.1)$ & $1 / 29(3.5)$ & $14 / 29(48.3)$ \\
\hline $2011(28 / I 7 I)$ & 0 & $7 / 28(25)$ & 0 & $14 / 28(50)$ & $2 \mathrm{I} / 28(75)$ \\
\hline $2012(32 / 17 \mid)$ & $4 / 32(12.5)$ & $13 / 32(40.6)$ & 0 & $6 / 32(18.8)$ & 23/32(71.9) \\
\hline $2013(34 /|7|)$ & II/34(32.4) & $8 / 34(23.5)$ & $3 / 34(8.8)$ & 0 & $22 / 34(64.7)$ \\
\hline $2014(\mid 5 / 171)$ & 0 & $7 / 15(46.7)$ & 0 & $5 / 15(33.3)$ & $12 / 15(80)$ \\
\hline $2015(20 / 171)$ & 0 & 0 & $5 / 20(25)$ & $4 / 20(20)$ & $9 / 20(45)$ \\
\hline $2016(|3 / 17|)$ & $5 / 13(38.5)$ & $2 / 13(15.4)$ & $1 / 13(7.7)$ & 0 & $8 / 13(61.5)$ \\
\hline Overall & $20 / 17 \mid(\mid 1.7)$ & $43 / 17 \mid(25.2)$ & $16 / 171(9.4)$ & $30 / 171(17.5)$ & $109 / 17 \mid(63.7)$ \\
\hline
\end{tabular}

\section{Temporal distribution of serotypes}

Outbreaks involving several serotypes occurred simultaneously during the study period. In addition, during the study period, there was at least one outbreak going on with at least one serotype involved. The line graph for serotype A shows three distinct outbreaks occurred i.e. July 2012- January 2013, in 2016 (January to July) and the end of 2016. The line graph for serotype $O$ shows that outbreaks occur at least once a year except for 2015. The line graph for serotype SAT -1 shows 4 distinct outbreaks occurring. These were in January 2010-January 2011, July 2013-January 2014, April 2015-January 2016 and JuneDecember 2016. The line graph for serotype SAT-2 show 3 distinct outbreaks 2010, 2011-2013 and 2014-2015 Figure 1.

\section{Effect of month of submission and seasonality}

A Generalized Linear Model was fitted against the log of the count of the number of samples submitted per month as the outcome. The predictor variable was the month. May was chosen as the base month since it had the lowest number of submitted samples according to best practice. The year of sampling and the sub county were also added to the model to correct for any clustering by year and sub county. Incidence Risk Rates (IRR) and their 95\% CI are shown in the table below. The relative risk for an increase in submitted suspected samples was highest in September and lowest in April. The two rainy seasons in the area generally occur in April to June and October-December. The highest was observed in the months before the beginning of a rainy season (at the end of the dry season) for each season i.e. March and September while the lowest relative risk was at the beginning of the rainy seasons i.e. April and October. The relative risk for an increase in positive samples was highest in November $(5.33$; 2.98 $9.56,95 \%$ CI $)$ and lowest in July $(1.33 ; 0.69-2.57,95 \%$ CI). There was no observed pattern for seasonality in positivity of samples except that during the dry season (July-September) the relative risk increased. The number of samples collected was significantly higher in the dry season than in the wet season $(\mathrm{t}(\mathrm{DF}=169)=2.30, \mathrm{p}=0.02)$.

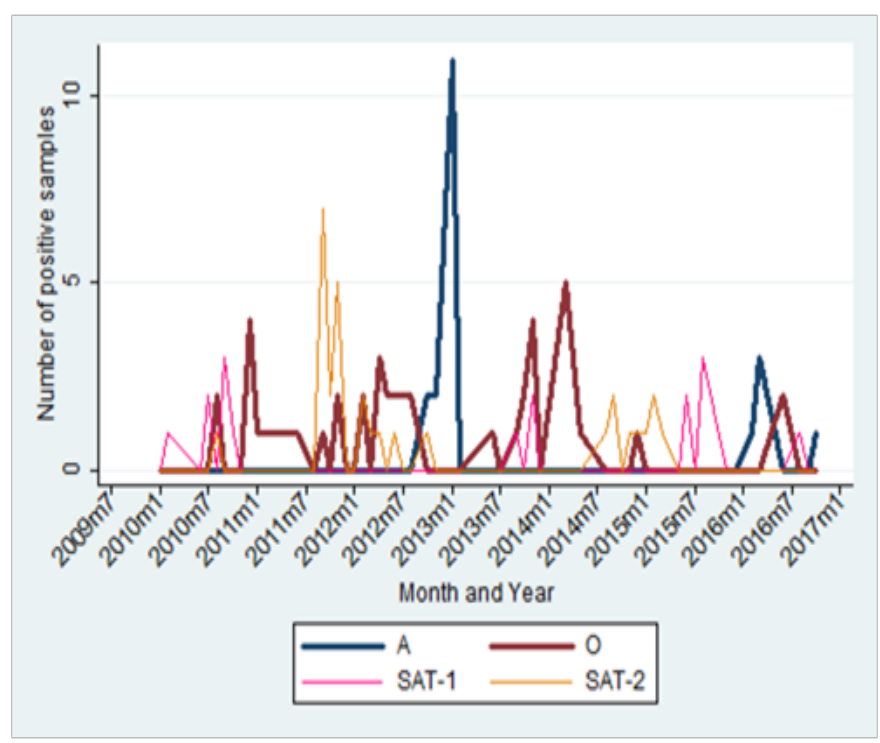

Figure I Combined line graph for all serotypes 2010-2016 in Nakuru county. 
Table 2 Results from a generalized linear model on monthly counts of submitted and positive samples

\begin{tabular}{lll}
\hline Month & Samples Submission & Sample Positivity \\
\hline & IRR(95\% CI) & IRR(95\% CI) \\
\hline January & $4.25(2.53-7.12)$ & $4.33(2.40-7.84)$ \\
February & $2.75(1.62-4.68)$ & $2.67(1.46-4.89)$ \\
March & $4.75(2.84-7.92)$ & $3.67(2.04-6.58)$ \\
April & $1.25(0.66-2.35)$ & $1.67(0.83-3.33)$ \\
May & Base month & Base month \\
June & $3.75(2.25-6.26)$ & $3(1.66-5.44)$ \\
July & $2.25(1.31-3.86)$ & $1.33(0.69-2.57)$ \\
August & $3.75(2.24-6.27)$ & $3.33(1.85-6.02)$ \\
September & $6.5(3.91-10.80)$ & $5(2.79-8.97)$ \\
October & $3.25(1.94-5.46)$ & $3(1.65-5.46)$ \\
November & $6(3.6 I-9.97)$ & $5.33(2.98-9.56)$ \\
December & $3.25(I .93-5.48)$ & $2(1.08-3.70)$ \\
\hline
\end{tabular}

\section{Discussion}

The yearly distribution of the samples was more or less equal except for 2014 and 2016 when fewer samples were collected. This might be related to fewer outbreaks occurring or due to some other factor leading to reduced reporting. Serotypes SAT 2 and $\mathrm{O}$ were the most isolated followed by A and SAT 1. This is consistent with findings by Sangula $\mathrm{AK}^{8}$ who found that $\mathrm{O}$ and SAT 2 were the most commonly isolated serotypes in the years 2004-2006 with A only causing intermittent outbreaks. Each year had at least two serotypes occurring with the combination of SAT 2 and $\mathrm{O}$ being the most common combinations. This agrees with findings by Sangula $\mathrm{AK}^{8}$ for the period 2004-2006. Serotype A was only isolated in 3years as compared to SAT 2 (5years) and O (6years). SAT 1 was isolated only once in 4years. This implies that SAT 2 and $\mathrm{O}$ are the most commonly occurring serotypes in the region. Temporal analysis shows that more than one serotype circulated at the same time in the region each year. Immunity to one serotype does not confer protection to a different serotype. Serotype A had 3 distinct outbreaks during the 6year period. This is consistent with findings by Sangula $\mathrm{AK}^{8}$ that this serotype only causes intermittent outbreaks. Serotypes O had outbreaks in each year under observation. Serotype SAT-1 had 4 distinct outbreaks while SAT 2 had 3. An outbreak was described as a cluster of cases occurring with no more than two months between them. However, without sequencing it would be hard to conclusively determine outbreaks since subtypes of the same serotype might be occurring simultaneously.

Serotype A had the biggest outbreak based on the number of positive samples perhaps because of an increase in susceptibility of the animals in the area due to the relatively rare occurrence of outbreaks with this serotype. There was some evidence of seasonality in sampling collection. FMD prevalence is thought to be higher in the dry seasons than in the rainy seasons coinciding with pastoralist movements in search of food and water. ${ }^{9}$ However this seasonality was weakly observed for positivity of the samples. Several factors might affect the positivity of laboratory samples including type of sample collected, handling of the samples, tests conducted and amount of pathogen material in the sample. The number of samples collected was significantly higher in the dry season compared to the wet season. This was probably because a greater number of FMD outbreaks are thought to occur in the dry season. Although this is some form of sampling bias, control of this is usually impossible for passive surveillance systems. Intermittent active surveillance activities can be scheduled in seasons of low incidence to complement the passive surveillance systems. ${ }^{10}$

\section{Conclusion}

Foot and Disease continues to be a major concern in Kenya with outbreaks in Nakuru County with outbreaks occurring yearly. In addition the outbreaks are caused by more than one serotype with outbreaks with different serotypes sometimes occurring simultaneously. There is weak evidence of seasonality in outbreak sampling and this should inform disease control measures. However this also leads to sampling bias due to the nature of the passive surveillance systems which may be mitigated by intermittent sampling during periods of low incidence. There is also need to investigate the role of pastoralist animal movements to spread of Foot and Mouth disease in Kenya.

\section{Acknowledgements}

None.

\section{Conflict of interest}

The authors declare that there is no conflict of interest.

\section{References}

1. Domingo E, Eric Baranowski Cristina Escarmís Francisco Sobrino. Foot-and-mouth disease virus. Comparative immunology, microbiology and infectious diseases. 2002;25(5-6):297-308.

2. Kibore B, Gitao CG, Sangula A, et al. Foot and mouth disease sero-prevalence in cattle in Kenya. Journal of Veterinary Medicine and Animal Health. 2013;5(9):262-268.

3. Lyons NA, Alexander N, Stärk KD, et al. Impact of foot-and-mouth disease on milk production on a large-scale dairy farm in Kenya. Prev Vet Med. 2015;120(2):177-186.

4. Vosloo W. Review of the status and control of foot and mouth disease in sub-Saharan Africa. Revue scientifique et technique. 2002;21(3):437-449.

5. Piriyawat $\mathrm{P}$, Smajsová M, Smith MA, et al. Comparison of Active and Passive Surveillance for Cerebrovascular Disease: The Brain Attack Surveillance in Corpus Christi (BASIC) Project. Am J Epidemiol. 2002;156(11):1062-1069.

6. Alice Namatovu, Sabenzia Nabalayo Wekesa, Kirsten Tjørnehøj, et al. Laboratory capacity for diagnosis of foot-and-mouth disease in Eastern Africa: implications for the progressive control pathway. BMC Veterinary Research. 2013;9(1):19.

7. Kibore B. Determination Of Foot-And-Mouth Disease Virus Seroprevalence. In Kenya Using The Liquid Phase Blocking And Nonstructural Protein Elisa Tests. 2013.

8. Sangula AK. Foot-and-Mouth disease serotypes SAT1 and SAT2 Epidemiology in East Africa. Food and Agriculture Organisation of the United Nations: Foot and Mouth Disease Laboratory. Kenya; 2006:1-8.

9. Rufael T, Catley A, Bogale A, et al. Foot and mouth disease in the Borana pastoral system, southern Ethiopia and implications for livelihoods and international trade. Tropical Animal Health and Production. 2008;40(1):29-38.

10. Naumova EN, Chen JT, Griffiths JK, et al. Use of passive surveillance data to study temporal and spatial variation in the incidence of giardiasis and cryptosporidiosis. Public Health Reports. 2000;115(5):436-447. 